\title{
217 - ECN Award: Effect of a Chronic Disease Self-Management Support Program for Spouse Caregivers of Relatives with Dementia in Shanghai, China: A Randomised Controlled Study
}

\author{
Author List : Xiaoshan Rong ${ }^{1}, \mathrm{PhD}$ candidate; Fan $\mathrm{Wu}^{1}, \mathrm{MM}$; Shuying Zhang ${ }^{*}, \mathrm{PhD}$ \\ 1Tongji University School of Medicine, Tongji University \\ *Corresponding authors
}

Background: Research found that caregivers of relatives with dementia (RWD) were more vulnerable to chronic diseases than those caring for relatives with other diseases. Unfamiliarity with and/or difficulty in balancing their own health needs was common among the caregivers, from the findings of our previous studies and relevant literatures. Therefore, an educational and psycho-social support program named Chronic Disease Self-Management Support (CD-SMS) program was developed for spouse caregivers of RWD in Shanghai, China.

Research Objective: This study was to examine the effectiveness of the CD-SMS program on spouse caregivers' health-related results, including caregiving self-efficacy, self-efficacy for chronic condition management, and their health behavior.

Method: This study was a randomised, wait-list controlled design. A total of 53 participants with common chronic conditions were recruited and assigned to experimental $(n=25)$ and waiting-list control group $(n=28)$ by stratified randomization. All participants received an illustrated psychoeducation booklet. The intervention group received six bio-weekly support group sessions, and the control group will receive the same support group sessions after the posttest. Participants in both groups received baseline and post-test assessments. The caregivers were assessed with the Chinese versions of Self-Efficacy for Managing Chronic Disease 6-item Scale, Self-Efficacy Questionnaire for Chinese Family Caregivers and Self-Management Behaviors Scale. The care recipients were measured with the Chinese version of Disability Assessment in Dementia.

Preliminary results of the ongoing study: A significant interaction between time and group was found in total scores of self-efficacy for managing chronic disease and caregiving self-efficacy for gathering information $(P<0.05)$. After intervention, caregivers in experimental group reported significant improvements in the total scores of self-efficacy for managing chronic disease, caregiving self-efficacy, and three self-management behaviors (stretching and strengthening exercise, cognitive symptom management, and communication with physicians). The care recipients' levels of activities of daily living in each group were worse at posttest compared to those at baseline $(P<0.01)$.

Conclusion: The findings indicated that the CD-SMS program was effective in improving the caregivers' confidence in balancing their caregiving management and their chronic health conditions management, and their main health behaviors were also improved.

Registration trial number: ChiCTR1800015602

Keywords: Dementia, Caregiver, Chronic disease, Self-management, Social support group. 